\title{
Bioactive Phytochemical Screening and Antioxidant Potential of Different Solvent Extracts of Anchote: The Underutilized Delicious Cultural Food of Oromo People, Ethiopia
}

\author{
Melkamu Biyana Regasa*, Kefyalew Gomoro Fayisa, Haftom Hagos Woldegebriel \\ Department of Chemistry, Wollega University, Ethiopia
}

Copyright $\bigcirc 2018$ by authors, all rights reserved. Authors agree that this article remains permanently open access under the terms of the Creative Commons Attribution License 4.0 International License

\begin{abstract}
Anchote is an indigenous tuberous crop that is commonly produced by Oromo nation in western and southwestern Oromia region in Ethiopia. It has nutritional, medicinal, economic and social importance. In the present study, attention is given to the medicinal values of Anchote since it is used culturally by the Oromo people for the treatment of many ailments like cancer, malaria, tuberculosis, gonorrhea and diabetes. The purpose of the present study is to test the presence of phytochemical compounds and evaluate the antioxidant potential of Anchote tuber. The samples were collected and extracted using water, methanol and diethyl ether sequentially and their phytochemical screening showed the presence of bioactive compounds such as reducing sugar, terpenoids, alkaloids, stereoids, tannins, saponins, phlobatannins and coumarins. All the extracts of Anchote showed maximum activities at the highest concentrations which might be due to the presence of phenols responsible for the radical scavenging activities observed. There is a significant correlation between the presence and the antioxidant activity at the half maximal inhibitory concentration (IC50) values on $\mathrm{DPPH}^{-}(\mathrm{P}<0.05)$. The medicinal value of Anchote may attribute to the presence of various polyphenols and the synergistic mixture of them. In conclusion, the presence of bioactive compounds in anchote tuber can be the evidence for its use for medicinal purposes. Therefore, its production in large scale, promotion and further study to isolate the potential antioxidant compounds are recommended to exploit its dual advantages (food and medicinal values).
\end{abstract}

Keywords Anchote, Antioxidant Activity, Cultural Food, Ethiopia, Phytochemicals, Oromo People

\section{Introduction}

Western and southwestern Oromia Regional state, Ethiopia is known by its production of edible plant (Ancootee-Afaan Oromoo, Anchote-English) which is considered as high valued cultural food among Oromo people on various occasions such as wedding, birthday, graduation ceremony, and holidays. Anchote [Coccinia abyssinica (Lam.) Cogn.] is an endemic plant grown for its edible tuberous roots in Ethiopia [1,2]. It is widely distributed and cultivated in the western and southwestern parts of Ethiopia especially in Jimma, Illu-Abba-Bora and Wollega Zones of the Oromia Regional State [3, 4]. It is a subsistence crop commonly produced to fill food security gaps during the hunger months (June to September). The production of Anchote has strong cultural ties with Oromo Nation, since it is used as cultural food during the finding of true cross locally called "Meskel Festival". It is an indigenous tuberous crop that is commonly produced by Oromo Nation in Ethiopia and later expanded to the Southern parts of Ethiopia to support the maintenance of food security in the region [5]. The total yield of Anchote is 150-180 quintals/hectare, which is in the range of the total yield of sweet potato, and potato [6]. This plant has nutritional, medicinal, economic and social importance and look to have its center of origin and diversity in the western and southwestern parts of Ethiopia in particular Wollega zones [7]. Anchote is among the root and tuber crops which have been the main components of the traditional foods of the southern, southwestern and western parts of Ethiopia. It is a good source of protein, carbohydrate, calcium and iron [2][8][9]. Traditionally, boiled after peeling or boiled before peeling and/ or further cooking are applied prior to consumption. Anchote is found both cultivated and wild [7]. Anchote is a valuable food source and according to 
local farmers, it helps in fast mending of broken/ fracture bones and displaced joints, as it contains high calcium, and proteins than other common and wide spread root and tuber crops [10]. By tradition, it is also believed that, Anchote makes lactating mothers healthier and stronger [11]. It was also reported that the juice prepared from tubers of Anchote has saponin as an active substance and is used to treat gonorrhoea, tuberculosis, malaria and cancer $[4,12]$. Wollega people use over stayed (over matured) Anchote tuber when they face a problem of bone-fracture and when women give birth, due to the fact that it is supposed to contain high $\mathrm{Ca}$ and $\mathrm{Fe}[13,14]$. In other words, the local people believe that Anchote has medicinal value in healing many maladies. Generally, it has nutritional (contains proteins, carbohydrate, $\mathrm{Ca}, \mathrm{Fe}, \mathrm{Zn}$ ), medicinal (used for the treatment of gonorrhea, tuberculoses, cancer, malaria etc), economic (good yield from small scale farm and used as a cash-crop) and social importance. A growing body of evidence indicates that secondary plant metabolites play critical roles in human health and may be nutritionally important [15]. Phytochemical screening of plants has revealed the presence of numerous chemicals including alkaloids, tannins, flavonoids, steroids, glycosides, saponins etc. Many plant extracts and phytochemicals show antioxidant or free radical scavenging properties due to the presence of phytochemicals $[16,17,18]$. Regardless of these facts, Anchote has not been subjected to scientific study to investigate the possible natural compounds contributing to the culturally believed of healing many diseases. Various plants (e.g. Anchote) contain secondary metabolites that are claimed to be used for the treatment of various diseases in many countries of the world including Ethiopia. So far, there is no scientific data on the phytochemical constituent and antioxidant activity of Anchote though it is used traditionally for various purposes including as a food source and medicinal values. Therefore, this study was designed for screening of bioactive plant compounds and the analysis of the related antioxidant potential of Anchote used in Wollega, Ethiopia.

\section{Methodology}

\subsection{Sample Collection}

The samples were purchased from local market in Nekemte town during the harvesting time of Anchote to obtain the uninfected matured sample in January 2017. A sub-total of about 3 kilograms of Anchote tuber samples were obtained from the bulk sample of 12 kilograms collected from local retailers in Nekemte town, East Wollega Zone, Ethiopia. The samples were packed in polyethylene bags, and transported to the Chemistry Laboratory, Wollega University. The sample was preserved for further use.

\subsection{Chemicals and Reagents}

All the chemicals and solvents used in the present study were of high purity (>99\%). Ascorbic acid (AA), methanol, distilled water, diethyl ether, hydrochloric acid, 1,1-diphenyl-2-picrylhydrazyl (DPPH), ammonia, chloroform, acetic acid, Mayer's reagent, Draggendorff's reagent, ferric chloride, sulfuric acid, olive oil, Fehling solution A, Fehling solution B, sodium hydroxide, isoamyl alcohol, benzene, and ammonium hydroxide.

\subsection{Sample Preparation}

The Anchote samples collected were washed in the laboratory by running tap water to remove soil and other foreign materials. The cleaned samples were cut into pieces; air dried and grinded using mortar and pestle. $50 \mathrm{~g}$ each of the powedered sample was taken into a separatory funnel and sequentially soaked with sufficient amount of water, methanol and diethyl ether, agitated periodically for 12 days respectively. The solution was then filtered with the help of filter paper and the filtered extract of the sample was dried using the rotatory vapour to near dryness under controlled condition of temperature and pressure. The extract residues were stored in deep freeze and used for preliminary phytochemical screening of secondary metabolites and to check the antioxidants activity used for analysis without further treatment $[19,20]$.

\subsection{Phytochemical Screening}

Phytochemical screening tests were done to evaluate the qualitative chemical composition of Anchote root crude extracts using commonly employed precipitation and coloration reactions to identify the major secondary metabolites like alkaloids, flavonoids, phenolic compounds, saponins, reducing sugars, steroids, tannins and terpenoids. For the extract of Anchote, phytochemical screening tests were made using the standard procedures reported by different scholars $[19,20,21,22]$.

\subsubsection{Testing the Presence of Alkaloids}

In order to test the presence or absence of alkaloids in all collected sample, $0.5 \mathrm{~g}$ of each solvent extract was diluted to $10 \mathrm{~mL}$ with acid alcohol (mixture of acid and alcohol), boiled and filtered. From this filtrate, $5.0 \mathrm{~mL}$ was taken and $2.0 \mathrm{~mL}$ of dilute ammonia was added to it. Then $5.0 \mathrm{~mL}$ of chloroform was added and shaken gently to extract the alkaloid base. The chloroform layer was extracted with $10.0 \mathrm{~mL}$ of acetic acid. This solution was divided into two portions and Mayer's reagent was added to the first and Draggendorff's reagent to the second one. The formation of a cream with Mayer's reagent or reddish brown precipitate with Draggendorff's reagent was regarded as positive for the presence of alkaloids. 


\subsubsection{Testing the Presence of Tannins}

From each extract sample, about $0.5 \mathrm{~g}$ was boiled in 10.0 $\mathrm{mL}$ of water in a test tube and then filtered. A few drops of $0.1 \%$ ferric chloride was added and observed for color change. The formation of brownish green or a blue black coloration indicates the presence of tannins in the plant material.

\subsubsection{Testing the Presence of Saponins}

Exactly $0.5 \mathrm{~g}$ of each extract was taken into a test tube and $5.0 \mathrm{~mL}$ of distilled water was added to it. The solution was shaken vigorously and observed for a stable persistent froth. The frothing was mixed with 3.0 drops of olive oil and shaken vigorously after which it was observed for the formation of an emulsion-indicates the presence of saponins in the plant sample.

\subsubsection{Testing the Presence of Flavonoids}

The presence of flavonoids in plants can be checked by many ways. In the present study, for the confirmation of the target compounds in the Anchote, $0.5 \mathrm{~g}$ of each extract were added in a test tube and $10.0 \mathrm{~mL}$ of distilled water, 5.0 $\mathrm{mL}$ of dilute ammonia solution were added to a portion of the aqueous filtrate of each extract followed by addition of $1.0 \mathrm{~mL}$ concentrated $\mathrm{H}_{2} \mathrm{SO}_{4}$ and the color change is observed. The formation of yellow color shows the presence of flavonoids in each plant extract.

\subsubsection{Testing the Presence of Terpenoids}

For this test, $0.5 \mathrm{~g}$ each extract was added $2.0 \mathrm{~mL}$ of chloroform. To these solutions, $3.0 \mathrm{~mL}$ of concentrated $\mathrm{H}_{2} \mathrm{SO}_{4}$ was carefully added to form a layer. A reddish brown colouration of the interface indicates the presence of terpenoids.

\subsubsection{Testing the Presence of Phlobatannins}

Anchote powder sample was mixed with distilled water in a test tube, then shaked it well, and filtered to take the extract. Then to each Anchote extract, $1 \%$ aqueous hydrochloric acid was added and each plant sample was then boiled with the help of Hot plate stirrer. The formation of red colored precipitate confirmed a positive result-presence of phlobatannins in the plant sample.

\subsubsection{Testing the Presence of Reducing Sugars (Fehling's Test)}

An amount of $0.5 \mathrm{~g}$ of powedered sample was added in $5.0 \mathrm{~mL}$ of distilled water and $1.0 \mathrm{~mL}$ of ethanol was mixed. After that we have taken $1.0 \mathrm{~mL}$ of Fehling solution A and $1.0 \mathrm{~mL}$ of Fehling solution B into different test tubes, heated it to boiling and then poured it in the aqueous ethanol extract obtained above. The observation of color reaction was regarded as a confirmatory test for the presence of reducing sugars in the plant sample extract.

\subsubsection{Testing the Presence of Coumarins}

$5.0 \mathrm{~g}$ of the powdered sample was taken into $25.0 \mathrm{~mL}$ of sterile water in borosilicate beaker, boiled at $50-60^{\circ} \mathrm{C}$ for 30 minutes on water bath and it was filtered through Whatman No.1 filter paper. Then the filtrate was centrifuged at $2500 \mathrm{rpm}$ for 15 minutes and filtrate was stored in sterile bottles at $5^{\circ} \mathrm{C}$ for further use. To check the presence or absence of coumarins in the plant extract, 3.0 $\mathrm{mL}$ of $10 \% \mathrm{NaOH}$ was added to $2.0 \mathrm{~mL}$ of aqueous extract and the formation of yellow colour indicates the presence of these phytochemicals.

\subsubsection{Steroids Test}

To test steroids, $1.0 \mathrm{~mL}$ of the extract was dissolved in $10.0 \mathrm{~mL}$ of chloroform and equal volume of concentrated sulphuric acid was added by sides of the test tube. The upper layer turns red and sulphuric acid layer showed yellow with green fluorescence. This indicated the presence of steroids.

\subsubsection{Anthocyanins Test}

To test anthocynins, $2.0 \mathrm{~mL}$ of aqueous extract is added to $2.0 \mathrm{~mL}$ of $2 \mathrm{~N} \mathrm{HCl}$ and ammonia. The appearance of pink-red turns blue-violet indicates the presence of anthocyanins.

\subsubsection{Leucoanthocyanins Test}

$5.0 \mathrm{~mL}$ of aqueous extract added to $5.0 \mathrm{~mL}$ of isoamyl alcohol. Upper layer appears red in colour indicates for presence of leucoanthocyanins.

\subsubsection{Emodins Test}

$2.0 \mathrm{~mL}$ of $\mathrm{NH}_{4} \mathrm{OH}$ and $3.0 \mathrm{~mL}$ of Benzene was added to the extract. Appearance of red colour indicates the presence of emodins.

\subsection{Determination of Antioxidant Activity by DPPH Method}

Antioxidant activity assay based on $\alpha$, $\alpha$-diphenyl- $\beta$-picrylhydrazyl (DPPH) free radical scavenging method offers the simplest approach for evaluating the antioxidant potential of a compound, an extract or other biological sources. It is based on the measurement of the scavenging capacity of antioxidants towards it. The odd electron of nitrogen atom in DPPH is reduced by receiving a hydrogen atom from antioxidants to the corresponding hydrazine.The radical scavenging activities of the plant extracts against DPPH radical (Sigma-Aldrich) were determined by UV spectrophotometry at $517 \mathrm{~nm}$. Radical scavenging activity was measured by a slightly modified method previously described. The effects of free radical scavenging activity by methanolic extracts of the plants were processed by DPPH method. To evaluate antioxidant activity, solution of $0.135 \mathrm{mM}$ DPPH (2, 2-Diphenyl-2-picryllhydrazyl) in methanol was prepared and $1.0 \mathrm{ml}$ of this solution was mixed with $1.0 \mathrm{~mL}$ of extract in methanol containing 
20-100 $\mu \mathrm{g}$ of the extract. The reaction mixture was vortexed thoroughly and incubated in the dark at room temperature for $30 \mathrm{~min}$. After 30 minutes of incubation, the discolouration of the purple color or the decrease in absorbance of the mixture was measured at $517 \mathrm{~nm}$ using Double beam UV-Vis Spectrophotometer (Thermo Electron Corporation, Cambridge, England). Ascorbic acid was used as reference standard. Methanol served as the blank and DPPH in methanol, without the extracts, served as the positive control. The antioxidant activity is calculated by determining the decrease in the absorbance at different concentration by using the equation.

$$
\begin{aligned}
& \text { Antioxidant activity }(\%)= \\
& (\text { Abs of control - Abs of sample) } \times 100 \%
\end{aligned}
$$

\section{Abs of control}

where Abs of control is the absorbance of mixture of DPPH radical, methanol, water and diethyl ether; Abs of sample is the absorbance of DPPH radical + sample extract/standard [23]. Measurements were taken at least in triplicate. The actual decrease in absorption induced by the test compounds was compared with the standards. $20.0 \mathrm{~mL}$ of the extract was placed in a beaker, and $3.0 \mathrm{~mL}$ of methanol was added followed by $0.5 \mathrm{~mL}$ of $0.1 \mathrm{mM}$ DPPH in methanol. A blank solution was prepared containing the same amount of methanol and DPPH. Lower absorbance of the solution indicates highest scavenging activity.

\subsection{Data Analysis}

Statistical Analysis of mean values and standard deviations was calculated from the results $(n=3)$ using analysis of variance (ANOVA) using SPSS version 20.0 for windows. Means were compared using Duncan's multiple range tests. The test of significance was applied as required and values obtained as $\mathrm{p}<0.05$ were regarded as significant statistically.

\section{Results and Discussion}

\subsection{Qualitative Screening of Major Phytochemicals}

The use of medicinal plants is directly dependent on the presence or absence of naturally occurring different kinds of biologically active substances. The utilization of plants in the treatment of certain human diseases is evidence of man's ingenuity. The contribution of these plants to the therapeutic arsenal in the fight against disease dates back several centuries, and has, to a certain extent, been documented by the ancient Chinese, Indian and North African civilizations. Currently, traditional medicine is widely practiced, especially in developing countries. In recent years, secondary plant metabolites like phytochemicals investigation have got better attention than ever. The qualitative phytochemical screening of the crude powder of Anchote root was done to assess the presence of bioactive components and the assay revealed the presence of different phytochemicals. Phytochemical screening test result of water, methanol and diethyl ether soluble fractions of Anchote tuber and the results were shown in Table 1. The phytochemical test for Anchote tuber results obtained indicated that the methanol extract contained reducing sugar, terpenoids, stereoids, tannins, saponnins and coumarins while water soluble fractions were detected for the presence of phlobatannins, reducing sugar, terpenoids, stereoids, saponnins, tannins and coumarins. Flavonoids, alkaloids, anthocyanins, leucoanthocyanins and emodins are absent in both methanol and water extracts however, except phlobatannins, all the other tested phytochemicals are present in methanol extract.

Table 1. Phytochemical screening of methanol, water and diethyl ether soluble fractions

\begin{tabular}{cccc}
\hline & \multicolumn{3}{c}{ Solvents } \\
\cline { 2 - 4 } Phytochemicals & Water & Methanol & $\begin{array}{c}\text { Diethyl } \\
\text { ether }\end{array}$ \\
\hline Phlobatannins & + & - & - \\
Reducing sugars & + & + & + \\
Terpenoids & + & + & + \\
Flavonoids & - & - & - \\
Alkaloids & - & - & + \\
Steroids & + & + & + \\
Tannins & + & + & - \\
Saponins & + & + & - \\
Anthocyanins & - & - & - \\
Leucoanthocyanins & - & - & + \\
Coumarins & + & + & - \\
Emodins & - & + & - \\
\hline
\end{tabular}

Key: "." indicates absence, "+” indicates presence

\subsubsection{Phlobatannins}

These group fo phytochemicals were detected only in water while the soluble fractions of methanol and diethyel showed negative results-the absenece of phlobatannins. The appearance of red color precipitate ensured the presence of phlobatannins in anchote tuber in the current study. The present result indicates that water can remove alkaloids from plants' samples than methanol and diethyl ether.

\subsubsection{Reducing Sugars}

For the three solvents, reducing sugar was found to be present. This implies all of the extracts were checked to contain reducing sugars. During this test, the observation of colored reaction was considered for the indication of the presence of reducing sugar in the extract. Reducing sugar may attribute to the usage of Anchote as a source of energy among Oromo people during different occasions.

\subsubsection{Terpenoids}

Terpenoids were detected in $100 \%$ of the extracts of the 
three solvents (methanol, water and diethyl ether) used in the present study. The presence of terpenoids was inferred from the formation of the reddish brown between the two layers of sulphuric acid and chloroform added to the solution of the extract. The result indicates that Anchote can be a god source of terpenoids which is very important in treating certain diseases. As such the cultural medicinal value of Anchote by Oromo people may be due to the presence of such the biologically active natural plant componds in it.

\subsubsection{Flavonoids}

Based on the qualitative assay of Anchote root extract for flavoids, all the extracts of methanol, water and diethyl ether extracts were detected for the absence of flavonoids. In the present work, the absence of yellow color formation up on the addition of concentrated sulphuric acid to the extract solution indicated the absence of flavonoids. This may attribute to the complete absence or very low concentration of flavonoids in the extracts. The most important biological activities like anticancer activity and anticancer agents believed by Anchote producers may be due to the presences of other phenolic compounds [24,25].

\subsubsection{Alkaloids}

The presence of alkaloids was detected only in diethyl ether soluble fractions of Anchote root. The actual observation of the reddish brown color and the cream formation during the addition of Draggendorff's reagent and Mayer's reagent indicates the presence of alkaloids. Alkaloids have been used as antiprotozoal, cytotoxic, antidiabetic and anti-inflammatory [26,27]. The existence of these phytochemicals makes anchote the possible alternative medicinal food for solving the disease protection problems observed among different community. The farmers cultivating anchote use it for dual purposes-food source and medicinal value which is reasonable based the present result.

\subsubsection{Stereoids}

Stereoids were detected in $100 \%$ of the extracts of the three solvents (methanol, water and diethyl ether) used in the present study. The observation of the turning of the upper layer of the solution to red and lower layer to yellow with green fluorescence upon the addition of chloroform and concentrated sulphuric acid to the extract's solutions confirmed the presence of stereoids.

Plant stereoids are known to be important for their cardiotonic activities, possession of insecticidal, anti-inflammatory, analgesic properties, central nervous system activities and antimicrobial properties [28]. Furthermore, they are used in nutrition, herbal medicine and cosmotics [29]. The result indicates that Anchote can be a source of stereoids which is very important in treating certain diseases. As such the cultural medicinal value of Anchote by Oromo people may be due to the presence of such a biologically active natural plant componds in it.

\subsubsection{Tannins}

Based on the chemical structures there are two groups of tannins. These are hydrolysable tannins (polymers of ellagic acid, or of gallic and ellagic acids, with glucose) and condensed tannins, which result from the condensation of monomers of flavan-3-ol units. Tannins are substances that are able to combine with proteins of animal hide preventing their putrefaction and converting them into leather. This ability comprises all kinds of proteins and, therefore, enzymes are included [30,31].

In the present study, the screening for tannins, the anchote root soluble fractions of methanol and water soluble fractions shows its presence. The formation of brownish green color in this study was regarded as an indication of the presence of these target chemicals. However, the diethyl ether extract showed negative result-the absence of saponins. Water and methanol extracts were detected for the presence while diethyl ether was tested for the absence of tannins. The pharmacological properties of tannins include antidiabetic, anti-inflammatory, antibacterial, antitumor activities and inhibition of HIV replication [28]. Tannins from active plant extracts are thought to be responsible for antidiarrhoeal activity by increasing colonic water and electrolyte reabsorption. The idea that says anchote can be used to treat many diseases like diabete is inline with this result-the presence of tannins.

Tannins exert their antioxidant activity by scavenging free radicals, chelating trace metals and by binding proteins with suppression of their enzymatic activity [32]. Yokozawa and his colleagues showed that the scavenging activity of tannins increases with an increase in the number of galloyl groups and molecular weight and in the presence of an ortho-dihydroxy structure: the hydroxyl groups are responsible for the chelating and radical scavenging properties of these compounds [33]. These compounds also showed $[34,35]$ have described the capacity of tannins to enhance glucose uptake and inhibit adipogenesis, thus being potential drugs for the treatment of non-insulin dependent diabetes mellitus. The antioxidant properties of tannins and flavonoids are equally responsible for other interesting biological properties. In particular, proanthocyanidins and flavan-3-ol monomers aid in lowering plasma cholesterol levels, inhibit low density lipoproteins (LDL) oxidation, and activate endothelial nitric oxide synthase to prevent platelet adhesion and aggregation that contribute to blood clot formation $[25,36]$.

\subsubsection{Saponins}

For screening saponnins, the actual observation of an emulsion was used to test the presence or absence of saponins. The test for saponins in the current study indicates that methanol and water soluble fractions shows its presence. However, the diethyl ether extract showed 
negative result-the absence of saponins while water and methanol extracts were only detected for the presence of saponins. Saponins are among the most abundant natural compounds in plants used as a mild detergents and in intracellular histochemistry staining to allow antibody access to intracellular proteins. Its medicinal uses are due to its properties like hypercholesterolemia, hyperglycemia, antioxidant, anticancer, anti-inflammatory, central nervous system activities[27] and weight loss as well antifungal [28].

\subsubsection{Anthocyanins}

The multiple possibilities regarding the identity and position of sugars and acyl moieties, as well as the position and number of hydroxy and methoxy groups on the anthocyanidin skeleton, gives rise to a great number of compounds, with over 600 anthocyanins being known today [37]. For the anchote extract, the change from pink-red to blue violet was considered for the positive result in the current research work. The qualitative assay of anthocyanins indicates that the Anchote tuber extracts of methanol, water and diethyl ether do not contain the target phytochemical. This may attribute to the complete absence or very low concentration of anthocyanins in the extracts. Further method development and optimization is required to justify this point.

In the past few years, more attention has been given to the study of adducts between anthocyanins and several other compounds, such as organic acids, either natural-occurring or synthetic. An unusual $C$-glycosyl-anthocyanin has been described by Tatsuzawa and colleagues from the flowers of the toad lily, Tricyrtis formosana (Liliaceae) which is the source of C-glycosylanthocyanins. Recently, significant anti-cancer properties of some anthocyanins against a range of cell lines have been described $[38,39,40]$.

\subsubsection{Leucoanthocyanins}

In this study the formation of red color was not observed at the upper layer of solution indicating the absence of leucoanthocyanins. The qualitative assay of leucoanthocyanins reveales that the Anchote root extracts of methanol, water and diethyl ether do not contain the target bioactive compound. This may attribute to the complete absence or very low concentration of leucoanthocyanins in the extracts.

\subsubsection{Coumarins}

Nowadays, around 1300 coumarins are known, with all of them being derivatives of 5, 6-benzo-2-pirone ( $\alpha$-chromone) (with $\mathrm{OH}, \mathrm{OCH}_{3}$ or $\mathrm{CH}_{3}$ substituents on the benzoic ring.). In addition to simple coumarins, $C$-prenylated and $O$-prenylated forms exist. In this study, coumarins were detected in the water, methanol and diethyl ether extracts. The result indicates that Anchote can be a source of coumarins which is very important in treating certain diseases. As such, the cultural medicinal value of Anchote by Oromo people may be due to the presence of such an important biologically active natural plant componds in it. As derivatives of simple coumarins, other compounds are known, such as furanocoumarins, which include a furanic ring, linear pyranocoumarins, angular pyranocoumarins, dimeric coumarins, of which dicoumarol is an example and also furanochromones [41]. Historically, the ability of dicoumarol to inhibit blood clotting, that later led to the development of the anticoagulant drug warfarin, was the first call to this class of compounds' biological properties. Several biological activities have been reported in natural-occurring coumarins, from photo sensitizers to vasodilatation. Recently, the interest has been given to synthetic derivatives of coumarins, such as fluorinated and 1-azo coumarins, which displayed moderate analgesia properties and excellent anti-inflammatory and anti-microbial activities which attracted the interest of many researchers to study the chemistry coumarins and their interesting biological and pharmacological properties [42].

\subsubsection{Emodins}

Based on the qualitative assay of emodins, Anchote tuber extracts of methanol, water and diethyl ether do not contain this target bioactive compound. This may attribute to the complete either the absence or very low concentration of emodins in the extracts.

\subsubsection{Antioxidant Activity Test}

Antioxidant activity determination help us to know the antioxidant content and their efficacy in foods, for preservation or protection against oxidative damage, to avoid deleterious changes and loss of commercial and nutritional value. Antioxidants of plant extracts have recently been of great interest in both research and the food industry because their possible uses as natural additives emerged from a growing tendency to replace synthetic antioxidants with natural ones as revealed by many research works. The antioxidant properties of plant extract is attributed to their polyphenolic contents and plants with high level of these chemicals have a greater importance as natural antimicrobics [43]. Many plants produce a huge amount of antioxidant and they can represent a potential source of new compounds having antioxidant properties [44].

In the present study, 2,2-Dipenyl-Picrylhydrazil (DPPH) radical scavenging method was used to investigate the antioxidant activity of methanol, water and diethyl ether soluble fractions obtained from the anchote plant species tubers. A rapid, simple and inexpensive method to measure antioxidant capacity of food involves the use of the free radical, 2,2-Diphenyl-1-picrylhydrazyl (DPPH). DPPH is widely used to test the ability of compounds to act as free radical scavengers or hydrogen donors, and to evaluate antioxidant activity of foods. It has also been used to 
quantify antioxidants in complex biological systems in recent years. The DPPH method can be used for solid or liquid samples and is not specific to any particular antioxidant component, but applies to the overall antioxidant capacity of the sample. A measure of total antioxidant capacity helps understand the functional properties of foods. Absorbance of each solution was measured at $517 \mathrm{~nm}$ and the percent inhibition were recorded to determine total antioxidant activity (Fig. 1).The percentage inhibition of DPPH free radical by water, methanol and diethyl ether extracts/ ascorbic acid at 517 $\mathrm{nm}$ were determined based on the mean value \pm standard deviation for triplicate measurements $(\mathrm{n}=3$ at $\mathrm{p}<0.05)$. Typical phenolics that possess antioxidant activity have been characterized as phenolic acids and flavonoids. Phenolic acids have repeatedly been implicated as natural antioxidants in fruits, vegetables, and other plants. In the present study the antioxidant activity test was found to be positive for each extract which can be attributed to the presence of phenols as shown in the phytochemical screening test result (Table 1). The comparative study shows that the antioxidant activity of water, methanol and diethyl ether extract for Anchote are comparable $(\mathrm{P}<0.05)$ i.e no significance difference is observed. The results obtained were comparative to standrads used DPPH and ascorbic acid.

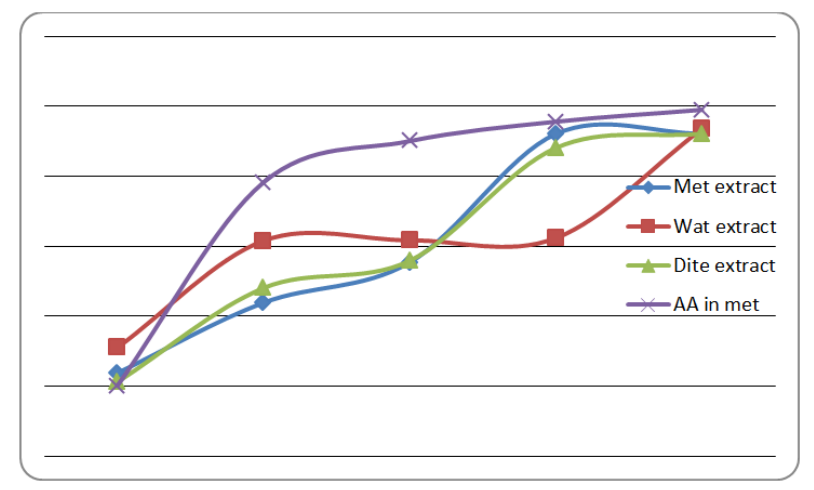

Met $=$ methanol, Wat $=$ water, Dite $=$ diethylether, $\mathrm{AA}=$ ascorbic acid

Figure 1. Comparison of $\%$ inhibition of water, methanol and diethyl ether extracts

The highest antioxidant activity (\% inhibition) values were observed for ascorbic acid (vitamin $\mathrm{C}$ ) which has recognized capacity of radical scavenging activity. The antioxidant potential of Anchote may be due to the three solvents soluble fractions containing various types of phenolic compounds which put synergistic effects in the radical scavenging activities. Polyphenols [45] and flavonoids [46] are reported to have significant antioxidant properties which lead to antioxidant activity [47]. Total phenolics possess a broad spectrum of chemical and biological activities including radical scavenging properties. The medicinal effects of plants are often attributed to the antioxidant activity of phytochemical constituents mainly phenolics, flavonoids and flavonols
[44]. It is reported that phenolic compounds are powerful chain breaking antioxidants [48]. The antioxidant activity has been reported to be concomitant with the development of reducing power [49]. Herbal preparation revealed synergistic effects both in DPPH scavenging and reducing power in comparison with the individual plant extracts selected for the study. The crude extracts of plants are pharmacologically more active than their isolated active principles due to the synergistic effects of various components present in the whole extract [50,51]. A statistically significant correlation was found between half maximal inhibitory concentration $\left(\mathrm{IC}_{50}\right)$ values on $\mathrm{DPPH}^{\text {. }}$ and $\mathrm{IC}_{50}$ values on oxygen radical scavenging activity $(\mathrm{r}=$ $0.9, \mathrm{P}<0.05)$. A correlation between reducing activity and the presence of mixed phenolic compounds was found to be positive and statistically significant $(r=0.94, \mathrm{P}<0.05)$. The maximum antioxidant potential of Anchote $(92 \%)$ is greater than that of T. Purthenium (80\%), A. Grandifolia (85\%), A. Crithmifolia (88\%), A. Absinthium (90\%), L. Latifolium (89\%), A.panicii (79\%), A. Sylvestris (36\%), H.officinalis (84\%)[52], Baccopa monnieri (82\%)[53], Withania somnifer Dunal (83\%)[54], Tacca pinatifida (76.53\%)[55], C. esculenta corm (19.35\%)[56], Dioscorea pentaphylla L.(89.41\%)[57] but lower than that of Centella asiatica (95.78\%)[53]. This work reveales that Anchote has significant antioxidant activity due to the availability of different phytochemicals in it. Maximum antioxidant potential of Anchote and other medicinal plants in terms of \% inhibition were compared (Table 2).

Table 2. Comparison of Anchote antioxidant potental with other medicinal plants

\begin{tabular}{|c|c|c|}
\hline Plant name & \% inhibition & \multirow{2}{*}{ Reference } \\
\hline T. Purthenium & 80 & \multirow{2}{*}{} \\
\cline { 1 - 2 } A. grandifolia & 85 & \multirow{2}{*}[52]{} \\
\cline { 1 - 2 } A. crithmifolia & 88 & \multirow{2}{*}{} \\
\hline A. absinthium & 90 & \\
\hline L. latifolium & 89 & \multirow{2}{*}[53]{} \\
\hline A. panicii & 79 & \\
\hline A. sylvestris & 36 & {$[54]$} \\
\hline H.officinalis & 84 & {$[55]$} \\
\hline Baccopa monnieri & 82 & {$[56]$} \\
\hline Centella asiatica & 95.78 & {$[57]$} \\
\hline Withania somnifer Dunal & 83 & Present work \\
\hline Tacca pinatifida & 76.53 & \\
\hline C. esculenta corm & 19.35 & \\
\hline Dioscorea pentaphylla L. & 89.41 & \\
\hline Anchote & 93 & \\
\hline
\end{tabular}

The antioxidant activity of these traditional medicinal plants may come in part from antioxidant vitamins, phenolics or tannins. Tannins and anthocyanins could also be factors in the antioxidant activities found in medicinal plants. Tannin itself is a strong antioxidant and is an especially prominent component in some of medicinal plants [58-60]. Antioxidants may act as free radical scavengers, reducing agents, chelating agents for the 
transition of metals, quenchers of singlet oxygen molecules and or activators of antioxidative defense enzyme system to suppress the radical damages in biological systems [61]. The reducing capacity of a compound may serve as an important indicator of its potential antioxidant activity. The reducing properties are generally associated with the presence of reductones [61].

\section{Conclusions}

In the present study, qualitative phytochemical analysis and antioxidant potential test of Anchote tuber were done. The extracts of the three solvents contain bioactive compounds such as phlobatannins, reducing sugar, terpenoids, alkaloids, stereoids, tannins, saponins and coumarins but donot cotain flavonoids, anthocyanins, leucoanthocyanins and emodins. Water extract was found to contain high phytochemical constituent while diethyl ether extract has low value. The medicinal value of Anchote may attribute to the presence of various polyphenols which showed strong antioxidant potential. As such, further study is strongly demanded to isolate, characterize and elucidate the structure of the bioactive compounds/antioxidants and their mode of actions which are responsible for its medicinal value. Furthermore, this cultural food is highly recommended to play its roles in food security confirmation attempts since it is rich in fibers, vitamins, minerals and other important compounds.

\section{Acknowledgements}

The authors are grateful to the Wollega University for its financial support in the form research and development project as well as material support including chemistry laboratory.

\section{Declaration}

The authors would like to declare that there is no conflict of interest among them.

\section{REFERENCES}

[1] Yosef, Y.; Tileye, F. (2013). Micropropagation of anchote [Coccinia abyssinica (Lam.) Cogn.]: High calcium content tuber crop of Ethiopia. African Journal Agricultural Research. 8(46), pp.5915-5922.

[2] Amare, G. (1973). Developmental anatomy of tubers of Anchote: potential dry land tuber crop. Acta Hort. No. 33.

[3] Fufa, H.; Urga, K. (1997). Nutritional and anti-nutritional characteristics of Anchote (Coccinia abyssinica) Ethiop. J. Health Dev. 11(2):163-168.
[4] Getahun, A. (1969). Developmental Anatomy of Seedlings and Tuber of Anchote, Coccinia abyssinica (Cucurbitaceae). $\mathrm{PhD}$ Thesis, University of Florida.

[5] Girma, A. and Dereje, H. (2015). Yield and nutrient concentration of Anchote [Coccinia abyssinica (Lam.) Cogn.] affected by harvesting dates and in-situ storage. African Journal of Crop Science. 3(5):156-161.

[6] IAR (1986). Department of Horticulture. Roots and Tubers team progress report for the period 1978/79. Addis Ababa. 1986:1-9.

[7] Edwards, S. (1991). Crops with wild relatives in Ethiopia. In: Engels JMM, Hawkes JG, Melaku Worede (eds) Plant Genetic Resources of Ethiopia. Cambridge University Press, Cambridge, pp. 42-47.

[8] PGRC/E (Plant Genetic Resources Center of Ethiopia). (1988). IAR Newsletter. No. 18.

[9] Westphal, E. (1974). Pulses in Ethiopia, their taxonomy and agricultural significance. Center for Agricultural publishing and Documentation, Wageningen.

[10] Endashaw, B. (2007). Study on Actual Situation of Medical Plants in Ethiopia. Prepared for JAICAF (Japan Association for International Collaboration of Agriculture and Forestry), (2007) pp. 50-51.

[11] Abera, H. (1995). Anchote-An Endemic Tuber crop. Addis Ababa University, P.75.

[12] Dawit, A. and Estifanos, H. (1991). Plnts as a primary source of drugs in the traditional health practices of Ethiopia. In: Engels, J.M.M., Hawkes, J.G and Melaku Worede (eds.), Plant Genetic Resources of Ethiopia. Cambridge University Press.

[13] Abera, G. and Gudeta, H. (2007). Response of Anchote (Coccinia abyssinica) to Organic and Inorganic Fertilizers Rates and Plant Population Density in Western Oromia, Ethiopia. East Afr. J. Sci. (EAJS). 1 (2): 120-126.

[14] Yambo, Y.; Feyissa, T. (2013). Micropropagation of anchote (Coccinia abyssinica (Lam.) Cogn.]: High calcium content tuber crop of Ethiopia. Afr. J. Agric. Res. 8 (46):5915-5922.

[15] Hamberger, M. and Hastettman, K. (1991).Bioactivityinplants:thelinkbetweenphytochemistrya ndmedicine.Phytochemistry. 30:3864-3874.

[16] Larson, R.A. (1988). The antioxidants of higher plants. Phytochemistry 27: 969-978.

[17] Nair, S.; Li, W.; Kong, A.T. (2007). Natural dietary anti-cancer chemopreventive compounds: Redox-mediated differential signaling mechanisms in cytoprotection of normal cells versus cytotoxicity in tumor cells. Acta Pharmacologica Sinica 28: 459-472.

[18] Parekh, J.and Chanda, S. (2007a). In vitro antimicrobial activity and phytochemical analysis of some Indian medicinal plants. Turkish Journal of Biology 31: 53-58.

[19] Sofowora, A. (1993). Medicinal plants and traditional medicine in Africa. Spectrum Books, Indian; pp150.

[20] Trease, G.E. and Evans, W.C. (1989). Pharmacognosy. 13th edn. Bailliere Tindall, London, pp176-180.

[21] Birdi, T.; Daswani, P.; Brijesh, S.; Tetali, P.; Natu, A. 
(2010). Newer insights into the mechanism of action of Psidium guajava L. leaves in infectious diarrhea. BMC complement Altern Med 10:33.

[22] Rizk, A.M.(1982). Fitoterapia, 52:35-42.

[23] Mothana, R.A.; Lindequist, U.; Gruenert, R. and Bednarski, P.J. (2009). Studies of the In Vitro Anticancer, ntimicrobial and Antioxidant Potentials of Selected Yemeni Medicinal Plants from the Island Soqotra. BMC Complementary and Alternative Medicine, 9, 1- 11.

[24] Cushnie, T.P.T. and Lamb, A.J. (2005). Antimicrobial activity of flavonoids. Int. J. Antimicrob. Agents. 26: 343-356.

[25] Aron, P.M. and Kennedy, J.A.(2008). Flavan-3-ols: Nature, occurrence and biological activity. Mol. Nutr. Food Res. 52:79-104

[26] Akindele, A.J. and Adeyemi. O.O. (2007). Anti-inflammatory activity of the aqueous leaf extracts of Byrsocarpus coccineus. Fitoterapia, 78: 25-28.

[27] Malairajan, P.; Geetha, G.; Narasimhan, S.; and Veni, K.J. (2006). Analgesic activity of some Indian medicinal plants. Journal of Ethnopharmacology, 19: 425-428.

[28] Argal, A. and Pathak,A.K. (2006). CNS activity of Calotropis gigantea roots. Journal of Ethnopharmacology, 106: $142-145$.

[29] Moses, A.G.; Gatebe, E.; Gitu, L. and Rotich, H. (2013). Preliminary phytochemical screening of eight selected medicinal herbs used for the treatment of diabetes, amalria and pneumonia in Kisii region Kenya, European Journa of Applied Sciences,5(1):01-06.

[30] Bruneton, J. (1999). Pharmacognosie: phytochimie, plantes médicinales; Éditions Tec \& Doc: Paris.

[31] Waterhouse, A. (2002). Wine phenolics. Ann. N Y Acad. Sci. 957: 21-36.

[32] Seabra, R.M.; Andrade, P.B.; Valentão, P.; Fernandes, E.; Carvalho, F.; Bastos, M.L. (2006). In Biomaterials from Aquatic and Terrestrial organisms; Fingerman, M., Nagabhushanam, R., Eds.; Science Publishers: Enfield, NH, USA. pp. 115-174.

[33] Yokozawa, T.; Chen, C.P.; Dong, E.; Tanaka, T.; Nonaka, G.-I.; Nishioka, I.(1998). Study on the inhibitory effect of tannins and flavonoids against the 1,1-diphenyl-2-picrylhydrazyl radical. Biochem. Pharmacol. 56: 213-222.

[34] Muthusamy, V.S.; Anand, S.; Sangeetha, K.N.; Sujatha, S.; Lakshmi, B.A.B.S.(2008). Tannins present in Cichorium intybus enhance glucose uptake and inhibit adipogenesis in 3T3-L1 adipocytes through PTP1B inhibition. Chem. Biol. Interact. 174: 69-78.

[35] Liu, F.; Kim, J.; Li, Y.; Liu, X.; Li, J.; Chen, X. (2001). An extract of Lagerstroemia speciosa L. has insulin-like glucose uptake-stimulatory and adipocyte differentiation-inhibitory activities in 3T3-L1 cells. J. Nutr. 131: 2242-2247.

[36] Bagchi, D.B.; Sen, C.K.; Ray, S.D.; Das, D.K., Bagchi, M.; Preuss, H.G.; Vinson, J.A. (2003). Molecular mechanisms of cardioprotection by a novel grape seed proanthocyanidin extract. Mutat. Res. 523: 87-97.

[37] Anderson, O.M. (2002). Anthocyanin occurrences and analysis. In Proceedings of the International Workshop on Anthocyanins: Research and Development of Anthocyanins, Adelaide, South Australia, April 17-19.

[38] Mateus, N.; Oliveira, J.; Pissarra, J.; González-Paramás, A.M.; Rivas-Gonzalo, J.C.; Santos-Buelga, C.; Silva, A.M.S.; de Freitas, V. (2006). A new vinylpyranoanthocyanin pigment occurring in aged red wine. Food Chem. 97: 689-695.

[39] Tatsuzawa, F.; Saito, N.; Miyoshi, K.; Shinoda, K.; Shigihara, A.; Honda, T. (2004). Diacylated 8Cglucosylcyanidin 3-glucoside from the flowers of Tricyrtis formosana. Chem. Pharm. Bull.52:631- 633.

[40] Zhang, Y.; Seeram, N.P.; Lee, R.; Feng, L.; Heber, D. (2008). Isolation and identification of strawberry phenolics with antioxidant and human cancer cell antiproliferative properties. J. Agric. Food Chem. 56: 670-675.

[41] Cunha, A.P., Fitoquímica, F.; Gulbenkian, F.C.: Lisbon, Portugal, 2005.

[42] Kalkhambkar, R.G.; Kulkarni, G.M.; Kamanavalli, C.M.; Premkumar, N.; Asdaq, S.M.B.; Sun, C.M. (2008). Synthesis and biological activities of some new fluorinated coumarins and 1-aza coumarins. Eur. J. Med. Chem. 43: 2178-2188.

[43] Leung, I.K., Su, Y., Chen, R., Zang, A., Huang, Y., Chen, Z.Y.(2000). The flavins in black and catechins in green tea are equally effective antioxidants. Journal of Nutrition; 131:2248-2251.

[44] Miliauskas, G., Venskutonis, P.R., Van Beek, T.A. (2004). Screening of radical scavenging activity of some medicinal and aromatic plant extracts. Food Chemistry; 85:231-237.

[45] Toda, J., Khurana, M.; Ohinishi, M. (1991). Effect of phenol carboxylic acid on superoxide anion and lipid peroxidation induced by superoxide anion. Planta Medica; $57: 8-10$.

[46] Faure, M.; Lissi, E.; Torres, R.; Videla, L.A. (1991). Antioxidant activities of lignans and flavonoids. Phytochemistry; 29:3773-3775.France.

[47] Dong, Z. (2003). Molecular mechanisms and the chemopreventive effect of reveeretrol. Mutation Research; 523:145-150.

[48] Shahidi, Wanasundara P.D. (1992). Phenolic antioxidants Critical Review of Food Science and Nutrition, 32:67-103.

[49] Akinmoladun, A.C.; Ibukun, E.O.; Afor, E.; Akinrinlola, B.L.; Onibon, T.R.; Akinboboye, A.O.; Obutor, E.M.; Farombi, E.O. (2007). Chemical constituents and antioxidant activity of Alstonia boonei. African Journal of Biotechnology; 6:1197-1201.

[50] Hamberger, M.; Hastettman, K. (1991). Bioactivity in plants: the link between phytochemistry and medicine. Phytochemistry; 30:3864-3874.

[51] Hertog, M.G.L.; Feskens, E.J.M.; Kromhout, D.; Hertog, M.G.L.; Hollman; P.C.H.; Hertog, M.G.L.; Katan, M.B. (1993). Dietary antioxidant flavonoids and risk of coronary heart disease: the Zutphen Elderly study. The Lancet 342: 
$1007-1011$.

[52] Stankovic, N., Krstev, T.M., Zlatkovic, B., Jovanovic, V.S., Mitic, V., Jovic, J., Comi, L., Kocic, B., Bernsteine, N. (2015). Antibacterial and Antioxidant Activity of Traditional Medicinal Plants from the Balkan Peninsula, NJAS-Wageningen J. Life Sci. (2015), http://dx.doi.org/10.1016/j.njas.2015.12.006.

[53] Meena, H., Pandey, H.K., Pandey, P., Arya, M.C., Ahmed, Z.(2012). Evaluation of antioxidant activity of two important memory enhancing medicinal plants Baccopa monnieri and Centella asiatica. Indian Journal of Pharmacology. 44(1): 114-17.

[54] Ansari, A.Q., Ahmed, S.A., M. A. Waheed, M.A. and Juned A.S.(2013). Extraction and determination of antioxidant activity of Withania somnifera Dunal. European Journal of Experimental Biology. 3(5):502-507.

[55] Jagtap, S. and Satpute, R. (2014). Phytochemical Screening and Antioxidant Activity of Tuber Extracts of Tacca pinnatifida. International Journal of Recent Trends in Science and Technology. 9(3): 389-396.

[56] Yadav, M., Kushawaha, D.K., Chatterji, S. and Watal, G (2017). Assessment of antioxidant activity and phytochemical screening of colocasia esculenta corm. International journal of pharmaceutical sciences and research. 8(4): 1758-1764.

[57] Kumar, S., Mahanti, P., Singh, N.R., Rath, S.K., Jena, P.K., Patra, J.K. (2017). Antioxidant activity, antibacterial potential and characterization of active fraction of Dioscorea pentaphylla L. tuber extract collected from Similipal Biosphere Reserve, Odisha, India. Brazilian Journal of Pharmaceutical Sciences. 2017;53(4):e17006

[58] Fortier, G., 1949. Antidiabetic properties of Rhus typhina. Laval Medical 14, 477- 506.

[59] Arnason, T., Hebda, R.J., Johns, T., 1981. Use of plants for food and medicine by Native Peoples of eastern Canada. Canadian Journal of Botany 59, 2189-2325.

[60] Haslam, E., 1996. Natural polyphenols (vegetable tannins) as drugs: possible modes of action. Journal of Natural Products 59, 205-215.

[61] Zanwar, A.A., Hegde, M.H. and Bodhankar, S.L. (2011). Cardioprotective activity of flax lignin concentrate extracted from seeds of L. usitatissimum in isoprenalin induced myocardial necrosis in rats. Interdisciplinary Toxicology 4: 90-97.

[62] Ho, Y.L., Huang, S.S., Deng, J.S., Lin, Y.H., Chang, Y.S. and Huang, G.H. (2012). In vitro antioxidant properties and total phenolic contents of wetland medicinal plants in Taiwan. Botanical Studies 53: 55-66. 